\title{
COVID-19 : Current and Potential Therapeutic Approaches
}

\author{
Asmaa Khalifa ${ }^{1}$, Mai Ghoneim², and Bassma $\mathrm{Ali}^{3}$ \\ ${ }^{1}$ Pharos University Faculty of Pharmacy and Drug Manufacturing \\ ${ }^{2}$ Faculty of Pharmacy, University of Sadat City \\ ${ }^{3}$ Arab Academy for Science Technology and Maritime Transport
}

July 2, 2020

\begin{abstract}
Severe acute respiratory syndrome corona virus 2 (SARS-CoV-2), a new virus from Corona viruses family, was detected in China in December 2019. The virus was suddenly and vigorously disseminated among individuals allover the world hence the control on the virus was lost and COVID-19 pandemic was announced. Scientists start to screen all the available options to treat this newly evolved virus. Till now there is no validated treatment for SARS-CoV-2 patients and all drugs are under clinical investigation. This mini-review represents a summarized insight for the pathophysiology and the available SARS-CoV-2 therapy with the clinical trials associated with each drug category including antivirals, monoclonal antibodies, anticoagulants, convalescent plasma therapy, miscellaneous and adjuvant therapies.
\end{abstract}

COVID-19: Current and Potential Therapeutic Approaches

\section{Asmaa A. Khalifa, PhD (1), Mai El-Sayed Ghoneim, ${ }^{\left(S^{(2)}\right.}$, Bassma Mahmoud Ali, MS ${ }^{(3)}$}

1. Department of Pharmacology and therapeutics, Faculty of Pharmacy and drug manufacturing, Pharos University, Alexandria, Egypt

2. Department of Pharmacology and toxicology, faculty of Pharmacy, University of Sadat City, Menofia, Egypt

3. Department of Special Chemistry, Arab Academy for Sciences and Technology and Maritime Transport, Alexandria, Egypt

Contact details of Corresponding author :

Name: Asmaa Ahmed Khalifa

Email : asmaa.khalifa@pua.edu.eg

Phone number: +0201274832271

By the end of 2019, some local health authorities reported significant number of patients with unkown cause pneumonia. However, all patients have a common factor that they where in a market in Wuhan, China. On January 2020, the WHO announced that COVID-19 caused by severe acute respiratory syndrome coronavirus-2 (SARS-COV-2) is a pandemic disease (Sanders et al., 2020). This virus belongs to coronaviruses single-stranded RNA family which can infect both animals and humans. Till now there is no standard treatment, however, massive research efforts were made to manage this novel virus. To present the therapeutic approches that are currntly avilable, the understanding of the pathophysiology of the virus is a must.

\section{Pathophysiology of the virus:}


SARS-CoV-2 has four structural proteins, which are "S" for spike, "E" for envelope, "M" for membrane, and "N" for nucleocapsid, which encapsulates single-stranded viral RNA ( Sanders et al., 2020). Spike protein is cleaved into S1 and S2 by the host cell protease, one of which is transmembrane protease serine-2 (TMPRSS2) which requires certain $\mathrm{pH}$ for being active. S1 subunit binds with the host cell surface receptors, while S2 subunit mediates membrane fusion (Sanders et al., 2020). Spike protein has a strong binding affinity to human ACE-2 receptors which expressed in the respiratory system and likely uses them as a mechanism for cell entry (Zhang et al., 2020a). Then the viral genetic material is released into the host cell, the genomic RNA of coronavirus acts as mRNA for translation of the replicase polyprotein 1a and 1ab (Zhang et al., 2020a). Afterwards, autoproteolytic cleavage of these polyproteins produces number of non-structural proteins including RNA-dependent RNA polymerase, helicase and nonstructural protein 3, 4, and 6 . These nonstructural proteins are thought to be responsible for anchoring the coronavirus replication/transcription complex through recruitment of intracellular endoplasmic reticulum membranes to form double membrane vesicles (DMV). RNA-dependent RNA polymerase (RdRp) and helicase localize to DMV and drive the production of subgenomic RNAs from which the structural and accessory proteins are produced in the next phase of translation (Chen et al., 2020). Once synthesized, transmembrane structural proteins "S", "M", and "E" are inserted, and folded in the ER and then transported to the endoplasmic reticulum-golgi intermediate compartment (ERGIC). The "N" proteins on contrast bind the viral genomic RNA in cytoplasm to form nucleocapsid. After assembly, mature virions are released by exocytosis to infect another cell.

\section{Therapeutic options of SARS-COV-2:}

\section{Antiviral drugs}

2. Remdesivir:

Remdesivir, a monophosphate prodrug that metabolized to an active C-adenosine nucleoside triphosphate analogue, is shown to be the most promising and hopeful anti-viral therapeutic (Al-Tawfiq et al., 2020). The active metabolite of remdesivir works by targeting viral ( $\mathrm{RdRp}$ ) while evading proofreading by viral exoribonuclease, resulting in premature termination of viral transcription (Al-Tawfiq et al., 2020). In a cohort study of hospitalized patients for severe COVID-19 who were treated with remdesivir, the clinical enhancement was observed in $68 \%$ of patients (Grein et al., 2020).

\section{Favipiravir}

A guanine analogue prodrug, selectively inhibits RNA-dependent RNA polymerase, has been approved to be effective in the treatment of influenza and Ebola viruses. Favipiravir acts as a substrate of viral RNA polymerase and halting viral replication (Wang et al., 2020). Lately, clinical studies have demonstrated Favipiravir to have promising influence in treatment of Chinese patients with SARS-CoV-2 infection in terms of disease development and viral clearance (Cai et al., 2020).

\section{Lopinavir/ritonavir}

Lopinavir/Ritonavir is a combination of antiretroviral protease inhibitors used in contemporary treatment of chronic human immunodeficiency virus infection (Sanders et al., 2020). Ritonavir is combined with Lopinavir to increase its half-life of Lopinavir via inhibition of cytochrome P450. Several retrospective and non-randomized cohort studies ascertain that Lopinavir/Ritonavir could inhibit SARS-CoV-2 replication in lung (Kumar et al., 2020; Yao et al., 2020). However, a recent study has noticed that the efficacy of Remdesivir was superior to that of Lopinavir/Ritonavir (Sheahan et al., 2020).

\section{Umifenovir (Arbidol):}

Arbidol is a non-nucleoside antiviral and immunomodulating agent that was commonly used for influenza treatment in Russia and China. For SARS-CoV-2, Umifenovir can potentially target S protein/ACE2 interaction and inhibit membrane fusion of the viral envelope (Sanders et al., 2020). A clinical study showed that Umifenovir could accelerate and enhance the process of viral clearance, improve chest radiologic images, and reduce the demand for oxygen therapy in hospitalization (Xu et al., 2020). However, the drug is only available in few countries. 


\section{Monoclonal Antibodies (Sarilumab and Tocillzumab)}

It was demonstrated that SARS-CoV-2 is associated with "Cytokine storm" in which release of large amounts of inflammatory cytokines such as interleukin-6 (IL-6) occur. The increased pulmonary inflammatory response may result in increased alveolar-capillary gas exchange, making oxygenation difficult in patients with severe illness (Coperchini et al., 2020). Interleukin-6 inhibitors may ameliorate severe damage to lung tissue caused by cytokine storm in patients with serious COVID-19 infections (Zhang et al., 2020b).

Sarilumab and Tocillzumab are IL-6 receptor blockers used for treatment of rheumatoid arthritis (June and Olsen, 2016). Both drugs are associated with serious infections rendering the patients at risk of death from these serious infections. In addition, Tocillzumab may cause rare but serious cases of liver injury, and in some cases liver transplantation may be required (June and Olsen, 2016). Sarilumab had an off label use to act against severe advanced cases of COVID-19, however, the drug is still under clinical investigation without any current approval from FDA (Sanofi, 2020). Chinese trial found that COVID-19 patients experienced rapidly reduced fever and lower need for supplemental oxygen within days of receiving Tocillzumab (Campochiaro et al., 2020). China recently updated its COVID-19 treatment guidelines and approved the use of IL-6 inhibitors to treat patients with severe or critical disease and who developed cytokine storm (Sanofi, 2020).

\section{Anticoagulants:}

Coagulopathy was found in a significant number of COVID-19 non survivors. Coagulation laboratory tests are essential to be known for all COVID-19 patients (e.g: D-dimer, prothrombin time, and platelet count). It is considered that anticoagulant therapies is accompanied with decreased mortality in severe COVID-19 patients (Tang et al., 2020). The use of heparin (Low molecular weight heparin LMWH or unfractionated heparin UFH) has potential benefit over other anticoagulants due to its anticoagulant, anti-inflammatory and potentially anti-viral properties and its effects against ARDS (Shi et al., 2020). A retrospective clinical study has demonstrated the use of low molecular weight heparin as potential therapeutic agent for treatment of COVID-19 through mitigate the cytokine storm (Shi et al., 2020). Furthermore, LMWH or UFH are preferred in critically ill patients because of their shorter half-lives and fewer drug-drug interactions compared with oral anticoagulants (Tang et al., 2020).

\section{Hydroxychloroquine:}

Hydroxychloroquine is an antimalarial agent and used in treatment of rheumatoid arthritis and systemic lupus erythematosus. It blocks viral entrance into cells by inhibiting glycosylation of host receptors, endosomal acidification, and proteolytic processing. Moreover, has immunomodulatory effects through inhibition of cytokine production, autophagy, and lysosomal activity in host cells (Devaux et al., 2020). It has been reported that co-administration of azithromycin with hydroxychloroquine in resulted in superior virologic clearance in comparison with hydroxychloroquine monotherapy (Gautret et al., 2020). Hydroxychloroquine may cause rare but dangerous adverse effects, including retinopathy, hypoglycemia, and neuropsychiatric effects and further clinical trials are required to detect the safest and most effective dose required for the clearance of the new virus (Kalil, 2020).

\section{Convalescent plasma (CP) therapy:}

Objectives reports for convalescent plasma have been stated as salvage therapy in severe acute respiratory syndrome (SARS) and pandemic influenza H1N1. Plasma from recovered COVID-19 patients contains large quantity of antibodies capable of neutralizing SARS-CoV-2 and eradicating the pathogen from blood circulation and pulmonary tissues (Cantore and Valente, 2020). In the meantime, clinical symptoms and radiological examination rapidly improved within 7 days (Duan et al., 2020). However, the administration of CP may need more randomized clinical trials to compare the outcomes of treatment group with control one and to ensure the clinical benefits alone or in combination with other drugs.

\section{Miscellaneous agents}

2. 


\section{Teicoplanin}

Teicoplanin is a semisynthetic glycopeptide antibiotic that significantly inhibits SARS-CoV-2. It acts on an early stage of the viral life cycle by inhibiting the low-pH required for cleavage of the viral spike protein. Evidence about Teicoplanin showed that there is a problem about the effective dose required to inhibit the virus and more studies are required. (Zhang et al., 2020c).

\section{Camostat mesylate}

It is used for the treatment of pancreatitis in Japan and also was found to prevent SARS-CoV-2 cell entry in vitro through inhibition of the host serine protease, TMPRSS2. This novel mechanism provides an additional drug target for future research (Hoffmann et al., 2020).

Corticosteroidsreducelunginflammatoryresponses, whichmayleadtoARDS.However, theadverseeffectsmaysurpassthisbenefit,ran 19patient(Russelletal.,2020).

\section{Immuneenhancementtherapy}

Type1interferonshaveasignificantantiviralactivity invitroandarecurrentlyeffectiveagainstSARS-

CoVinclinicaltrials.Ithasbeendemonstratedthatinterferon1 $\beta$ maybeasafeandeasyfortreatmentagainstCOVID-

19intheearlystagesofinfection.SimilartherapieshadefficiencyagainstSARS-CoVviruses;however, invitrostudiesproposethatSARS CoV-2couldbemarkedlymoresensitivetointerferon1thanothercoronaviruses(Sallardetal.,2020). Thecombinationofinterferon1wit

\section{Melatonin}

Melatoninhasimmuneenhancing,anti-inflammationandanti-oxidantfeatures.Priorresearcheshavedocumentedthepotentialeffects 19patients(Zhangetal.,2020d). Inconclusion,thereisnostandardtherapyforallpatientsinfectedwithSARS-CoV-

2.Further,clinicaltrialsarerequiredforallavailableoptionstomakesurefromeffectiveness,safetyandproperdosesrequiredtofightagair $\mathrm{CoV}-2$

\section{REFERENCES:}

Al-Tawfiq, J. A., Al-Homoud, A. H., \& Memish, Z. A. (2020). Remdesivir as a possible therapeutic option for the COVID-19. Travel medicine and infectious disease, 34 , 101615.https:// doi:10.1016/j.tmaid.2020.101615.

Cai, Q., Yang, M., Liu, D., Chen, J., Shu, D., Xia, J. . . . \& Liu, L. (2020). Experimental Treatment with Favipiravir for COVID-19: An Open-Label Control Study. Engineering (Beijing) , 10.1016/j.eng.2020.1003.1007.https:// doi:10.1016/j.eng.2020.03.007.

Campochiaro, C., Della-Torre, E., Cavalli, G., De Luca, G., Ripa, M., Boffini, N. . . . \& Dagna, L. (2020). Efficacy and safety of tocilizumab in severe COVID-19 patients: a single-centre retrospective cohort study. European journal of internal medicine, 76 , 43-49.https:// doi:10.1016/j.ejim.2020.05.021.

Cantore, I., \& Valente, P. (2020). Convalescent plasma from COVID 19 patients enhances intensive care unit survival rate. A preliminary report. Transfusion and Apheresis Science, https://doi.org/10.1016/j.transci.2020.102848 .https://.

Chen, Y., Liu, Q., \& Guo, D. (2020). Emerging coronaviruses: Genome structure, replication, and pathogenesis. Journal of medical virology, 92 (4), 418-423.https:// doi:10.1002/jmv.25681.

Coperchini, F., Chiovato, L., Croce, L., Magri, F., \& Rotondi, M. (2020). The cytokine storm in COVID19: An overview of the involvement of the chemokine/chemokine-receptor system. Cytokine 85 growth factor reviews .https:// doi:10.1016/j.cytogfr.2020.05.003. 
Devaux, C. A., Rolain, J. M., Colson, P., \& Raoult, D. (2020). New insights on the antiviral effects of chloroquine against coronavirus: what to expect for COVID-19? International journal of antimicrobial agents, 55 (5), 105938.https:// doi:10.1016/j.ijantimicag.2020.105938.

Duan, K., Liu, B., Li, C., Zhang, H., Yu, T., Qu, J. . . . \& Hu, Y. (2020). Effectiveness of convalescent plasma therapy in severe COVID-19 patients. Proceedings of the National Academy of Sciences, 117 (17), 9490-9496.https://.

Gautret, P., Lagier, J. C., Parola, P., Hoang, V. T., Meddeb, L., Mailhe, M. . . . \& Raoult, D. (2020). Hydroxychloroquine and azithromycin as a treatment of COVID-19: results of an openlabel non-randomized clinical trial. International journal of antimicrobial agents , 105949.https:// doi:10.1016/j.ijantimicag.2020.105949.

Grein, J., Ohmagari, N., Shin, D., Diaz, G., Asperges, E., Castagna, A. . . . \& Lescure, F.-X. (2020). Compassionate use of remdesivir for patients with severe Covid-19.New England Journal of Medicine, 382 (24), 2327-2336.https://.

Hoffmann, M., Kleine-Weber, H., Schroeder, S., Krüger, N., Herrler, T., Erichsen, S. . . \& Pöhlmann, S. (2020). SARS-CoV-2 Cell Entry Depends on ACE2 and TMPRSS2 and Is Blocked by a Clinically Proven Protease Inhibitor. Cell, 181 (2), 271-280.e278.https:// doi:10.1016/j.cell.2020.02.052.

June, R. R., \& Olsen, N. J. (2016). Room for more IL-6 blockade? Sarilumab for the treatment of rheumatoid arthritis. Expert opinion on biological therapy, 16 (10), 1303-1309.https:// doi:10.1080/14712598.2016.1217988.

Kalil, A. C. (2020). Treating COVID-19-Off-Label Drug Use, Compassionate Use, and Randomized Clinical Trials During Pandemics. Jama .https:// doi:10.1001/jama.2020.4742.

Kumar, S., Zhi, K., Mukherji, A., \& Gerth, K. (2020). Repurposing antiviral protease inhibitors using extracellular vesicles for potential therapy of COVID-19. Viruses , 12 (5), 486.

Russell, C. D., Millar, J. E., \& Baillie, J. K. (2020). Clinical evidence does not support corticosteroid treatment for 2019-nCoV lung injury. Lancet (London, England), 395 (10223), 473-475.https://.

Sanders, J. M., Monogue, M. L., Jodlowski, T. Z., \& Cutrell, J. B. (2020). Pharmacologic Treatments for Coronavirus Disease 2019 (COVID-19): A Review. Jama, 323 (18), 1824-1836.https:// doi:10.1001/jama.2020.6019.

Sanofi. (2020). Sanofi and Regeneron begin global Kevzara(r) (sarilumab) clinical trial program in patients with severe COVID-19. Retrieved from http://www.news.sanofi.us/2020-03-16-Sanofi-and-Regeneron-beginglobal-Kevzara-R-sarilumab-clinical-trial-program-in-patients-with-severe-COVID-19

Sheahan, T. P., Sims, A. C., Leist, S. R., Schafer, A., Won, J., Brown, A. J. . . . \& Baric, R. S. (2020). Comparative therapeutic efficacy of remdesivir and combination lopinavir, ritonavir, and interferon beta against MERS-CoV. Nature communications, 11 (1), 222.https:// doi:10.1038/s41467-019-13940-6.

Sallard, E., Lescure, F. X., Yazdanpanah, Y., Mentre, F., Peiffer-Smadja, N., Florence, A. D. E. R., ... \& BOUADMA, L. (2020). Type 1 interferons as a potential treatment against COVID-19.Antiviral Research , 104791.

Shi, C., Wang, C., Wang, H., Yang, C., Cai, F., Zeng, F. . . . \& Deng, B. (2020). The potential of low molecular weight heparin to mitigate cytokine storm in severe COVID-19 patients: a retrospective clinical study. Medrxiv .https:// doi:10.1101/2020.03.28.20046144.

Tang , N., Bai , H., Chen, X., Gong, J., Li , D., \& Sun, Z. (2020). Anticoagulant treatment is associated with decreased mortality in severe coronavirus disease 2019 patients with coagulopathy. Journal of Thrombosis and Haemostasis , doi:10.1111/jth.14817.https://. 
Wang, M., Cao, R., Zhang, L., Yang, X., Liu, J., Xu, M. . . . \& Hu, Z. (2020). Remdesivir and chloroquine effectively inhibit the recently emerged novel coronavirus (2019-nCoV) in vitro. 30 (3), 269-271.https:// doi:10.1038/s41422-020-0282-0.

Xu, K., Yanfei, C., Yuan, J., Yi, P., Ding, C., Wu, W. . . . \& Zhao, H. (2020). Clinical Efficacy of Arbidol in Patients with 2019 Novel Coronavirus-Infected Pneumonia: A Retrospective Cohort Study. Journal of medical virology, http://dx.doi.org/10.2139/ssrn.3542148.https://.

Yao, T.-T., Qian, J.-D., Zhu, W.-Y., Wang, Y., \& Wang, G.-Q. (2020). A systematic review of lopinavir therapy for SARS coronavirus and MERS coronavirus-A possible reference for coronavirus disease-19 treatment option. Journal of medical virology, 92 (6), 556-563.https:// doi:10.1002/jmv.25729.

Zhang, H., Penninger, J. M., Li, Y., Zhong, N., \& Slutsky, A. S. (2020a). Angiotensin-converting enzyme 2 (ACE2) as a SARS-CoV-2 receptor: molecular mechanisms and potential therapeutic target. Intensive care medicine, 46 (4), 586-590.https:// doi:10.1007/s00134-020-05985-9.

Zhang, C., Wu, Z., Li, J. W., Zhao, H., \& Wang, G. Q. (2020b). Cytokine release syndrome in severe COVID-19: interleukin-6 receptor antagonist tocilizumab may be the key to reduce mortality. International journal of antimicrobial agents, 55 (5), 105954.https:// doi:10.1016/j.ijantimicag.2020.105954.

Zhang, J., Ma, X., Yu, F., Liu, J., Zou, F., Pan, T., \& Zhang, H. (2020c). Teicoplanin potently blocks the cell entry of 2019-nCoV. bioRxiv , 2020.2002.2005.935387.https:// doi:10.1101/2020.02.05.935387.

Zhang, R., Wang, X., Ni, L., Di, X., Ma, B., Niu, S. . . . \& Reiter, R. J. (2020d). COVID-19: Melatonin as a potential adjuvant treatment. Life sciences, 250 , 117583.https:// doi:10.1016/j.lfs.2020.117583. 